\title{
ON THE DISTRIBUTION OF SUMS OF RESIDUES
}

\author{
JERROLD R. GRIGGS
}

\begin{abstract}
We generalize and solve the $\bmod q$ analogue of a problem of Littlewood and Offord, raised by Vaughan and Wooley, concerning the distribution of the $2^{n}$ sums of the form $\sum_{i=1}^{n} \varepsilon_{i} a_{i}$, where each $\varepsilon_{i}$ is 0 or 1 . For all $q$, $n, k$ we determine the maximum, over all reduced residues $a_{i}$ and all sets $P$ consisting of $k$ arbitrary residues, of the number of these sums that belong to $P$.
\end{abstract}

\section{INTRODUCTION}

Vaughan and Wooley [15] raised the problem of determining the maximum number of the $2^{n}$ sums of the form $\sum_{i=1}^{n} \varepsilon_{i} a_{i}$, where each $\varepsilon_{i}$ is 0 or 1 , that are congruent to $0 \bmod q$. The maximum is over all residues $a_{1}, \ldots, a_{n}$ that are reduced, which means that $\left(a_{i}, q\right)=1$ for all $i$. Results about this problem have been applied to study the solutions of simultaneous additive equations.

By using analytical tools, including exponential sums and classical inequalities, and by treating many cases, Vaughan and Wooley show that the maximum is $\left(\begin{array}{c}n \\ \lfloor n / 2\rfloor\end{array}\right)$ provided that $q>\lceil n / 2\rceil$. This bound is sharp, since it is attained by letting $a_{i}$ be 1 for $i \leq\lceil n / 2\rceil$ and -1 for $i>\lceil n / 2\rceil$. (To see this, observe that for this choice of $a_{i}$ 's, we have $\sum_{i=1}^{n} \varepsilon_{i} a_{i} \equiv 0$ precisely when an equal number of $\varepsilon_{i}$ 's are 1 for $i \leq\lceil n / 2\rceil$ and -1 for $i>\lceil n / 2\rceil$. This happens if and only if the number of indices $i$ with $i \leq\lceil n / 2\rceil$ and $\varepsilon_{i}=1$ plus the number with $i>\lceil n / 2\rceil$ and $\varepsilon_{i}=0$ is $\lfloor n / 2\rfloor$, so that the choices correspond to the subsets of $\{1, \ldots, n\}$ of size $\lfloor n / 2\rfloor$.)

When $\lceil n / 2\rceil \geq q$, wraparound effects $\bmod q$ come into play. For example, with the $a_{i}$ 's chosen as above, the sum $\sum_{i=1}^{q} a_{i}$ is also congruent to 0 , so the answer exceeds $\left(\begin{array}{c}n \\ \lfloor n / 2\rfloor\end{array}\right)$.

We solve the problem for arbitrary $n$ and $q$, using an inductive argument that is inspired by the study of the extremal properties of the Boolean lattice $B_{n}$ on the collection $2^{[n]}$ of all subsets of the $n$-set $[n]=\{1, \ldots, n\}$, ordered by inclusion. Let us adopt the notation

$$
\left(\begin{array}{l}
n \\
s
\end{array}\right)_{q}:=|\{A \subseteq[n]:|A| \equiv s\}|=\sum_{j \equiv s}\left(\begin{array}{l}
j \\
s
\end{array}\right),
$$

for the $\bmod q$ binomial coefficients in $n$. We shall see that for general $n$ and $q$, the maximum number of sums congruent to 0 is the middle $\bmod q$ binomial coefficient $\left(\begin{array}{c}n \\ \lfloor n / 2\rfloor\end{array}\right)_{q}$. The maximum is attained as before by dividing

Received by the editors September 2, 1992.

1991 Mathematics Subject Classification. Primary 11P83; Secondary 11A07, 05A05, 06A07.

Research supported in part by NSA/MSP Grant MDA90-H-4028 and by a Visiting Professorship at Simon Fraser University. 
the $a_{i}$ 's as evenly as possible between 1 and -1 . In general, the maximum number of sums congruent to any single residue is $\left(\begin{array}{c}n \\ \lfloor n / 2\rfloor\end{array}\right)_{q}$. Throughout the paper we maintain the condition that the residues $a_{i}$ be reduced. Without this restriction, one would select $a_{i}$ 's with common factors, in order to increase the number of sums congruent to 0 .

This problem we are considering is the analogue for residues of a famous problem about the clustering of partial sums of a collection of complex numbers. In connection with their study of roots of random polynomials, Littlewood and Offord [13] were led to consider the following question. For $a_{1}, \ldots, a_{n} \in \mathbf{C}$ with $\left\|a_{i}\right\| \geq 1$ for all $i$ and for an open ball $S \subset \mathbf{C}$ of unit diameter, how many of the $2^{n}$ sums of the form $\sum_{i=1}^{n} \varepsilon_{i} a_{i}$, where each $\varepsilon_{i}$ is 0 or 1 , can belong to $S$ ? They sought the maximum over all choices of $a_{i}$ 's and $S$. In particular, if one selects $a_{i}$ to be 1 for all $i$ and centers $S$ at $\lfloor n / 2\rfloor$, one can pack $\left(\begin{array}{c}n \\ \lfloor n / 2\rfloor\end{array}\right)$ sums into $S$, and this was believed to be optimal. While Erdös [3] soon proved this for the real $a_{i}$ case, it was twenty years before the original complex case was solved, by Katona [8] and Kleitman [10] independently, from an appropriate extension of the theorem of Sperner [14] about the maximum size of an antichain in the Boolean lattice.

Although the usual Sperner method does not extend to higher dimensions, Kleitman [11, also in 5] found a remarkable proof that the answer is still $\left(\begin{array}{c}n \\ \lfloor n / 2\rfloor\end{array}\right)$ in any dimension $m$ (or indeed, in Hilbert space): For any vectors $a_{1}, \ldots, a_{n} \in \mathbf{R}^{m}$ of length at least one, there is a partition of $2^{[n]}$ into just $\left(\begin{array}{c}n \\ \lfloor n / 2\rfloor\end{array}\right)$ blocks, such that for any sets $I, J$ in the same block, the sums $\sum_{i \in I} a_{i}$ and $\sum_{j \in J} a_{j}$ are far apart (distance at least one). Hence any open ball $S$ of unit diameter contains at most $\left(\begin{array}{c}n \\ \lfloor n / 2\rfloor\end{array}\right)$ sums. The idea behind this construction is that for every $n$, the sizes of the blocks partitioning $2^{[n]}$ exactly match the sizes of the chains in the famous inductive symmetric chain decomposition of $B_{n}$ discovered by de Bruijn et al. [2].

Erdös [3] considered the more general problem of maximizing the number of sums of vectors inside an open ball in $\mathbf{R}^{m}$ of diameter $d \geq 1$. He solved this problem for the real case $(m=1)$ using Sperner theory, and he found that the value attained when all $a_{i}=1$ is optimal for all $d$. This value is the sum of the $\lceil d\rceil$ middle binomial coefficients, $\sum_{(n-\lceil d\rceil) / 2 \leq j<(n+\lceil d\rceil) / 2}\left(\begin{array}{l}n \\ j\end{array}\right)$. However, the problem is more complicated when $m \geq 2$ and completely solved only in some special cases. Using a variety of tools from extremal set theory, probability, and geometry, many authors have attacked this more general question, including Kleitman [12], Griggs [6], and Frankl and Füredi [4]. Also see the survey by Anderson [1].

In marked contrast to previous results of the Littlewood-Offord type, the setting for the work of Vaughan and Wooley is the additive group $\mathbf{Z}_{q}$ of integers $\bmod q$. Nonetheless, as with the unit diameter problem above, we shall see that their theorem can be obtained by an inductive partition construction inspired by a particular chain partition of the Boolean lattice. The method yields the solution to the extension of their problem to general $n$ and $q$.

More generally, we determine the maximum number of the $2^{n}$ sums $\sum_{i=1}^{n} \varepsilon_{i} a_{i}$ congruent $\bmod q$ to any of $k$ arbitrary residues $\rho_{j}$, for $1 \leq j \leq k$, 
over all choices of the residues $\rho_{j}$ and the reduced residues $a_{i}$. The answer is the sum of the $k$ middle $\bmod q$ binomial coefficients in $n$. This bound is attained by selecting all $a_{i}$ to be 1 and selecting the $k$ middle values for the residues $\rho_{j}$. Switching some $a_{i}$ in this solution to -1 has the effect of shifting the collection of all $2^{n}$ sums down by 1 . Thus the bound is also attained by selecting $a_{i}$ to be 1 for $i \leq\lceil n / 2\rceil$ and -1 for $i>\lceil n / 2\rceil$ and by choosing the $k$ initial values in the sequence $0,1,-1,2,-2, \ldots$ for the residues $\rho_{j}$.

\section{The MAIN Result}

We fix the integer $q>0$ and work in $\mathbf{Z}_{q}$.

Theorem 1. Let $a_{1}, \ldots, a_{n}$ be reduced residues in $\mathbf{Z}_{q}$. Let $P \subseteq \mathbf{Z}_{q}$, where $|P|=k$. Then the number of the $2^{n}$ sums $\sum_{i=1}^{n} \varepsilon_{i} a_{i}$ in $P$, where each $\varepsilon_{i}$ is 0 or 1 , is at most the sum of the $k$ middle $\bmod q$ binomial coefficients $\sum_{(n-k) / 2 \leq j<(n+k) / 2}\left(\begin{array}{l}n \\ j\end{array}\right)_{q}$, and this bound is best possible.

Proof. For $S \subseteq \mathbf{Z}_{q}$ and $a \in \mathbf{Z}_{q}$, let $S+a:=\{s+a: s \in S\} \subseteq \mathbf{Z}_{q}$. For $\mathscr{A} \subseteq 2^{[n]}$, define the sum set

$$
S(\mathscr{A})=\left\{\sum_{i \in I} a_{i}(\bmod q): I \subset \mathscr{A}\right\} .
$$

We say that $\mathscr{A} \subseteq 2^{[n]}$ is a structure for $a_{1}, \ldots, a_{n}$ provided that the sums in $S(\mathscr{A})$ are distinct.

We shall partition $2^{[n]}$ into $\left(\begin{array}{c}n \\ \lfloor n / 2\rfloor\end{array}\right)_{q}$ structures in such a way that the bound in the theorem will follow for all $k$. The construction is carried out by induction on $n$ for a given sequence of reduced residues $a_{1}, a_{2}, \ldots$. It starts at $n=0$ with the single structure $\{\varnothing\}$. For the induction step, suppose we are given a partition of $2^{[n-1]}$ into structures $\mathscr{A}_{j}$ for $a_{1}, \ldots, a_{n-1}$. Then the structures $\mathscr{A}_{j}$ and $\mathscr{A}_{j}^{\prime}:=\left\{I \cup\{n\}: I \in \mathscr{A}_{j}\right\}$ for $a_{1}, \ldots, a_{n}$ partition $2^{[n]}$, but they are not quite the ones we want. Notice that $S\left(\mathscr{A}_{j}^{\prime}\right)=S\left(\mathscr{A}_{j}\right)+a_{n}$. We require an easy fact.

Lemma. Let $\varnothing \neq S \subseteq \mathbf{Z}_{q}$ and $a \in \mathbf{Z}_{q}$ with $(a, q)=1$. Then $S+a=S$ if and only if $S=\mathbf{Z}_{q}$.

If $S\left(\mathscr{A}_{j}\right)$ is $\mathrm{Z}_{q}$, then so is $S\left(\mathscr{A}_{j}^{\prime}\right)$, and we leave both structures alone. However, if $S\left(\mathscr{A}_{j}\right) \neq \mathbf{Z}_{q}$, then by the lemma there exists at least one element $t \in S\left(\mathscr{A}_{j}^{\prime}\right) \backslash S\left(\mathscr{A}_{j}\right)$, say $t=\sum_{i \in I} a_{i}$ where $I \in \mathscr{A}_{j}^{\prime}$, so that we may replace $\mathscr{A}_{j}$ and $\mathscr{A}_{j}^{\prime}$ by the structures $\mathscr{B}_{j}=\mathscr{A}_{j} \cup\{I\}$ and $\mathscr{B}_{j}^{\prime}=\mathscr{A}_{j}^{\prime} \backslash\{I\}$. We have $\left|\mathscr{B}_{j}\right|=\left|\mathscr{A}_{j}\right|+1$ and $\left|\mathscr{B}_{j}^{\prime}\right|=\left|\mathscr{A}_{j}\right|-1$. In the case where $\left|\mathscr{A}_{j}\right|=1$, we discard $\mathscr{B}_{j}^{\prime}$.

Now denote the structures in this partition of $2^{[n]}$ by $\mathscr{A}_{j}$ for $j=1,2, \ldots$. Since sets in a structure have distinct sums, it follows that

$$
\left|\left\{I \subseteq[n]: \sum_{i \in I} a_{i} \in P\right\}\right| \leq \sum_{j} \min \left(k,\left|\mathscr{A}_{j}\right|\right) .
$$

It suffices to show that the sum on the right-hand side of inequality (1) is at most the sum of the $k$ middle $\bmod q$ binomial coefficients in $n$. 
Since the collection of structure sizes $\left|\mathscr{A}_{j}\right|$ depends in no way on the actual values of the $a_{i}$ 's, it is enough to consider the case where all $a_{i}=1$. One can verify by induction on $n$ that the sum set $S\left(\mathscr{A}_{j}\right)$ for each structure consists of all $q$ residues or else consists of values congruent to an interval $x$, $x+1, \ldots, y \in \mathbf{Z}$ centered about $n / 2$, which means $x+y=n$. The number of structures in the partition is $\left(\begin{array}{c}n \\ \lfloor n / 2\rfloor\end{array}\right)_{q}$, because every structure contains a set with sum (i.e., cardinality) $\equiv\lfloor n / 2\rfloor$. For general $k$, we see that the sum on the right-hand side of (1) is the number of subsets of cardinality congruent to any of the $k$ middle values around $n / 2$.

When $q>\lceil n / 2\rceil$, we have that $\left(\begin{array}{c}n \\ \lfloor n / 2\rfloor\end{array}\right)_{q}=\left(\begin{array}{c}n \\ \lfloor n / 2\rfloor\end{array}\right)$, which implies the original result of Vaughan and Wooley [15]:

Corollary 1. Let $a_{1}, \ldots, a_{n}$ be reduced residues in $\mathbf{Z}_{q}$, where $q>\lceil n / 2\rceil$. Then the number of the $2^{n}$ sums $\sum_{i=1}^{n} \varepsilon_{i} a_{i}$ congruent to 0 , where each $\varepsilon_{i}$ is 0 or 1 , is at most $\left(\begin{array}{c}n \\ \lfloor n / 2\rfloor\end{array}\right)$, and this bound is best possible.

\section{RELATED REMARKS}

The inspiration for the proof of the theorem is the inductive partition of the Boolean lattice $B_{n}$ into saturated chains of size at most $q$, that is, into collections of at most $q$ totally ordered subsets of consecutive sizes. Katona [9] used this construction to determine the maximum number of subsets of $\{1, \ldots, n\}$ containing no sets $A \subset B$ with $0<|B \backslash A|<q$. The author [7] later independently devised the same construction to obtain a maximumsized collection of disjoint saturated chains of size $q$ in $B_{n}$. The collection of structure sizes $\left|\mathscr{A}_{j}\right|$ in our construction exactly corresponds to the collection of chain sizes in Katona's partition.

By applying the theorem with $k=q-1$, it is also possible to determine the minimum number of sums in any residue class.

Corollary 2. Let $a_{1}, \ldots, a_{n}$ be reduced residues in $\mathbf{Z}_{q}$, where $n \geq q-1$. Let $\rho \in \mathbf{Z}_{q}$. Then the number of the $2^{n}$ sums $\sum_{i=1}^{n} \varepsilon_{i} a_{i}$ congruent to $\rho$, where each $\varepsilon_{i}$ is 0 or 1 , is at least $\left(\begin{array}{c}n \\ \lceil(n-q) / 2\rceil\end{array}\right)$, and this bound is best possible.

The bound in Corollary 2 is attained by taking all $a_{i}=1$ and $\rho \equiv\lceil(n-q) / 2\rceil$. For $n<q-1$, no sums are congruent to -1 when all $a_{i}$ equal 1 . The asymptotic growth of the $\bmod q$ binomial coefficients, studied in connection with saturated chain partitions [7], implies that the lower bound in Corollary 2 approaches $2^{n} / q$ as $n \rightarrow \infty$ with fixed $q$. (This remains true even if $q$ grows with $n$, provided that $q=o\left(n^{1 / 2}\right)$.) Hence, for any sequence $\left\{a_{1}, a_{2}, \ldots\right\}$ of reduced residues $\bmod q$, the distribution of the $\bmod q$ sums of the first $n$ residues is asymptotically uniform as $n \rightarrow \infty$.

The Littlewood-Offord problem has an equivalent formulation that considers the concentration of sums of the form $\sum_{i=1}^{n} \delta_{i} a_{i}$ with each $\delta_{i}=1$ or -1 , where as before $\left\|a_{i}\right\| \geq 1$ for all $i$. The analogous problem in $\mathbf{Z}_{q}$ can be solved by a reduction to the original problem of Theorem 1 . 
Corollary 3. Let $a_{1}, \ldots, a_{n}$ be reduced residues in $\mathbf{Z}_{q}$. Let $P \subseteq \mathbf{Z}_{q}$, where $|P|=k$. Then the number of the $2^{n}$ sums $\sum_{i=1}^{n} \delta_{i} a_{i}$ in $P$, where each $\delta_{i}$ is 1 or -1 , is at most the sum of the $k$ middle $\bmod r$ binomial coefficients in $n$, where $r$ is $q$ when $q$ is odd and $q / 2$ when $q$ is even, and this bound is best possible.

\section{ACKNOWLEDGMENT}

The author is grateful to Oren Patashnik and Ted Sweetser for many suggestions that greatly improved the presentation of this paper.

\section{REFERENCES}

1. I. Anderson, Combinatorics of finite sets, Clarendon Press, Oxford, 1987.

2. N. G. de Bruijn, C. A. van Ebbenhorst Tengbergen, and D. R. Kruyswijk, On the set of divisors of a number, Nieuw Arch. Wisk. (2) 23 (1952), 191-193.

3. P. Erdös, On a lemma of Littlewood and Offord, Bull. Amer. Math. Soc. 51 (1945), 898-902.

4. P. Frankl and Z. Füredi, The Littlewood-Offord problem in higher dimensions, Ann. of Math. (2) 128 (1988), 259-270.

5. C. Greene and D. J. Kleitman, Proof techniques in the theory of finite sets, Studies in Combinatorics (G.-C. Rota, ed.), Math. Assn. America, Philadelphia, PA, 1978, pp. 22-79.

6. J. R. Griggs, The Littlewood-Offord problem: Tightest packing and an M-part Sperner theorem, European J. Combin. 1 (1980), 225-234.

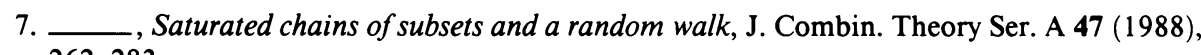
262-283.

8. G. O. H. Katona, On a conjecture of Erdös and a stronger form of Sperner's theorem, Studia Sci. Math. Hungar. 1 (1966), 59-63.

9. __ Families of subsets having no subset containing another with small difference, Nieuw Arch. Wisk. (3) 20 (1972), 54-67.

10. D. J. Kleitman, On a lemma of Littlewood and Offord on the distribution of certain sums, Math. Z. 90 (1965), 251-259.

11. On a lemma of Littlewood and Offord on the distributions of linear combinations of vectors, Adv. in Math. 5 (1970), 1-3.

12. __ Some new results on the Littlewood-Offord problem, J. Combin. Theory Ser. A 20 (1976), 89-113.

13. J. E. Littlewood and A. C. Offord, On the number of real roots of a random algebraic equation, Mat. Sb. 12 (1943), 277-286.

14. E. Sperner, Ein Satz über Untermengen einer endlichen Menge, Math. Z. 27 (1929), 544-548.

15. R. C. Vaughan and T. D. Wooley, On a problem related to one of Littlewood and Offord, Quart. J. Math. Oxford (2) 42 (1991), 379-386.

Department of Mathematics, University of South Carolina, Columbia, South CarOLINA 29208

E-mail address: griggs@math.scarolina.edu 